\title{
Medical Image Segmentation Based on Level Set Method
}

\author{
Md. Golam Moazzam ${ }^{1}$, Amita Chakraborty ${ }^{2}$, Shamima Nasrin ${ }^{2}$, and \\ Mohammad Selim ${ }^{2}$ \\ ${ }^{1}$ Department of Computer Science and Engineering, Jahangirnagar University, Bangladesh \\ ${ }^{2}$ Department of Computer Science and Engineering, Shaikh Burhanuddin College, Bangladesh
}

\begin{abstract}
This paper presents a shape-based approach to curve evolution for the segmentation of medical images. Automatic interpretation of medical images is a very difficult problem in computer vision. Several methods have been developed in last decade to improve the segmentation performance in computer vision. A promising mathematical framework based on variational models and partial differential equations has been investigated to solve the image segmentation problem. This approach benefits from well-established mathematical theories that allow people to analyze, understand and extend segmentation methods. In this paper, a variational formulation is considered to the segmentation using active contours models.
\end{abstract}

Keywords - Active Contour, Image Segmentation, Level Set Method, Morphological Erosion, Thresholding, Variational Level Set Method, Contour Evaluation.

\section{INTRODUCTION}

In computer vision image segmentation refers to the process of partitioning a digital image into multiple segments i.e. sets of pixels, also known as super pixels [1]. The goal of segmentation is to simplify and/or change the representation of an image into something that is more meaningful and easier to analyze. Image segmentation is typically used to locate objects and boundaries (lines, curves, etc.) in images. More precisely, image segmentation is the process of assigning a label to every pixel in an image such that pixels with the same label share certain visual characteristics. The result of image segmentation is a set of segments that collectively cover the entire image, or a set of contours extracted from the image. Each of the pixels in a region are similar with respect to some characteristic or computed property, such as color, intensity, or texture. Adjacent regions are significantly different with respect to the same characteristics. When applied to a stack of images, typical in Medical imaging, the resulting contours after image segmentation can be used to create 3D reconstructions with the help of interpolation algorithms like Marching cubes [1], [2].

Medical imaging is the set of digital image processing techniques that create and analyze images of the human body to assist doctors and medical scientists. In medicine, imaging is used for planning surgeries, X-ray imaging for bones, Magnetic resonance imaging, endoscopies and many other useful applications [3], [4].

Several general-purpose algorithms and techniques have been developed for image segmentation. Since there is no general solution to the image segmentation problem, these techniques often have to be combined with domain knowledge in order to effectively solve an image segmentation problem for a problem domain.

\section{PREVIOUS WORKS}

In computer vision literature, various methods dealing with segmentation and feature extraction are discussed. The well known technique of the morphological watershed transform creates a tessellation of the image domain in several small regions by considering the image values as intensity niveaus in a topographical landscape.

By simulating rainfall, the domain is grouped in catchment basins, regions in which the water drains from all points to the same local intensity minimum. Naturally, this method is very sensitive to small variations of the image magnitude and consequently the number of generated regions is undesirably large. To overcome this problem of identifying exhaustively many segments there have been investigated in recent years to reduce the complexity of the tessellations by region merging based on homogeneity criteria or studying the evolution of the catchment basins in Gaussian scalespace. Such techniques can generate unpredictable results and depend to a large extend on user interaction and the quality of the initial partition. Although improvements have been made, the creation of the watersheds is still computationally demanding [5].

An entirely popular approach to visual shape analysis is related to so called active contour models and snakes [6], [7], [8]. It is based on a curve respective surface evolution, starting from some initial curve or surface which is propagated to achieve a proper approximation of the segment boundary. Active contour models may incorporate a wide range of driving forces. Many of them are based on minimization of combined energy functionals controlling the fairness of the resulting curve on one hand and the attraction to areas of interest such as object boundaries on the other hand. Weighting parameters have to be carefully chosen to be a good balance 
between these terms. In early works explicit snakes with a standard parametric curve representation were used. The key disadvantage of this method is a topological constraint: the curve can not split to approximate boundaries of not simply connected segments. Such problems have been solved by introducing implicit snakes models [9], in which the initial curve is interpreted as the zero level curve of a function. The evolution of these snakes is controlled by a PDE [9]. An external term is considered to include information about the initial image. Although contours are able to split in this formulation, there remains the problem that the result of the segmentation relies significantly on a good initialization. Furthermore, many models have difficulties in progressing into boundary concavities. Addressing these particular problems a new class of external forces has been proposed by deriving from the original image a gradient vector in a variational framework [10]. Sensitivity to initialization has been drastically reduced and contours have a more sensible behavior in the regions of concavities.

\section{CONCEPT OF LEVEL SET METHOD}

The level set method is based on combination of several existing methods. Normally, the thresholding method is used to make an image binary. But the technique here is used in a new moderated way. Instead of making an image binary, a threshold value is defined to make all the value under threshold to 0 value and others to take the value as original image. This helps to keep original properties of original image and keep all value fixed as original image. Thus, further analysis will be based on original image. The main purpose of using this technique is to ignore unnecessary part of image that is not requiring for image segmentation. After applying thresholding technique, the image contains some small ignorable parts that also need to ignore. For this purpose, a morphological technique is used known as erosion. But in case of erosion a very small sized structured element is considered so that the original part of image is not removed. Finally, a new variational level set method is used to complete segmentation process. Instead of using traditional level set method, variational level set method is used to get better result. This variational process have some advantages, such as a significantly larger time step can be used for numerically solving the evaluation partial differential equation, and therefore, speed up the curve evaluation. Second, the level set function can be initialized with general functions that are more efficient to construct and easier to use in practice.

\section{PROPOSED METHOD}

In this paper, a novel segmentation method has been proposed to segment all types of images under a unique platform. For this reason, the most commonly used medical images of current world are employed as input. These images are not used in their original format. Instead, experiments have been performed successfully on the images of .JPG format. The main steps employed for new medical image segmentation technique based on Level Set Method is shown in Fig. 1.

\section{Thresholding}

The fundamental principle of thresholding techniques is based on the characteristics of the image. It chooses proper thresholds $\mathrm{T}$ to divide image pixels into several classes and separates the objects from background. When there is only a single threshold $T$, any point $(x, y)$ for which $f(x, y)>T$ is called an object point and a point $(\mathrm{x}, \mathrm{y})$ is called a background point if $\mathrm{f}(\mathrm{x}, \mathrm{y})<\mathrm{T}$ [11]. Here thresholding method has been used in a moderated way. Instead of making an image binary, a threshold value is defined to make all the value under threshold to 0 value and others to take the value as original image. It helps to determine the defected object more precisely. As thresholding technique has not been used in ordinary way, objects are determined within exact pixel value.

$$
g(x, y)= \begin{cases}f(x, y) & \text { if } f(x, y)>T \\ 0 & \text { if } f(x, y) \leq T\end{cases}
$$




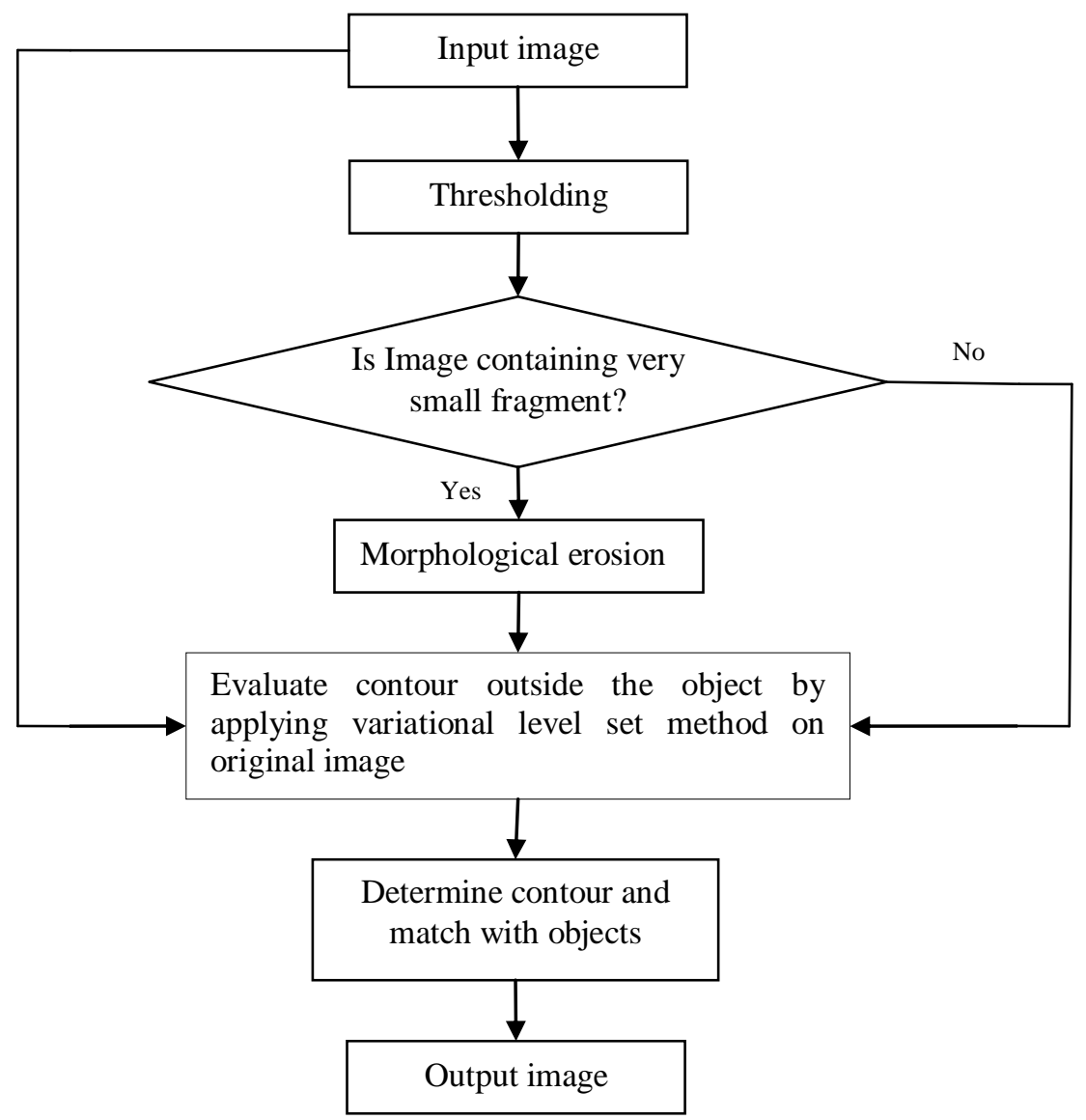

Fig. 1: Basic steps employed for new medical image segmentation technique based on level set method

In this method, the overall technique has been divided into several sub techniques. Thresholding is the most important among them and it is the first steps to segment the image more precisely. The given equation states that a threshold value $\mathrm{T}$ is chosen. The value under $\mathrm{T}$ is 0 and others are as like as original image $f(\mathrm{x}, \mathrm{y})$. As a result, it gives easily the required part. Fig. 2 demonstrates this process more precisely. A MR image is taken as input. Here the threshold value $\mathrm{T}$ is 201 and then it gives following output.

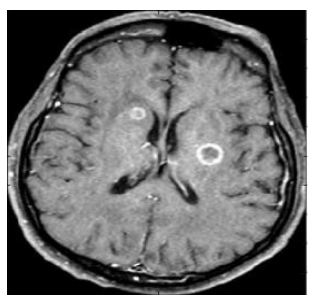

Fig. 2: Thresholding

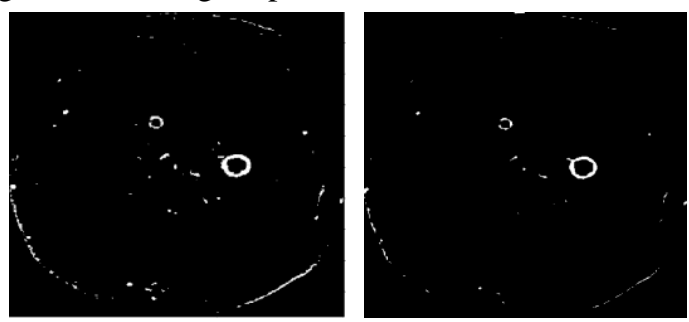

Fig. 3: Morphological Erosion

\section{Morphological Erosion}

The basic idea in binary morphology is to probe an image with a simple, pre-defined shape, drawing conclusions on how this shape fits or misses the shapes in the image. This simple "probe" is called structuring element, and is itself a binary image.

In this proposed technique the erosion process is used to remove unused or small part of image. The system will decide that either erosion technique is needed or not. This decision is taken according to image quality, how much the image is complex or how many small fragments are in the image after applying the thresholding technique. Basically, the erosion process is used to shrink an image. After applying the thresholding technique, the image contains some small ignorable parts that also need to ignore in practice [12]. For this purpose, a morphological technique is used known as erosion. But in case of erosion a very small sized 
structured element is considered so that the original part of image is not removed. Fig. 3 demonstrates this process more precisely.

\section{Variational Level Set Method}

The variational level set method is used to get better result. This variational process have some advantages, such as a significantly larger time step can be used for numerically solving the evaluation of partial differential equation and therefore, speed up the curve evaluation. Secondly, the level set function can be initialized with general functions that are more efficient to construct and easier to use in practice. In variational level set method, the image after erosion and the original image are used. The image after erosion or the image after thresholding can be used demanding on the quality of the image. If the image after applying morphological erosion is much complex or the image contains many small fragments in the thresholding technique, the variational technique is implemented using some steps. First, the image is smoothed using Gaussian Convolution. Then, edge indicator function is applied to the image after erosion or image after thresholding defending on the quality of the image. To achieve this goal, it explicitly defines an external energy that can move the zero level curve toward the object boundaries. Let $I$ be an image, and $g$ be the edge indicator function defined by,

$$
g=\frac{1}{1+\left|\nabla G_{\sigma} * I\right|^{2}}
$$

Then find coefficient of the internal (penalizing) energy term that help contour to outside the object boundary [10], [13]. It defines an external energy for a function $\emptyset(\mathrm{x}, \mathrm{y})$ as below:

$$
\varepsilon_{g, \lambda, v}(\phi)=\lambda l_{g}(\varphi)+v A_{g}(\varphi)
$$

where $\lambda>0$ and $v$ are constants, and the terms $\ell_{g}(\varphi)$ and $A_{g}(\varphi)$ are defined by,

$$
\ell_{g}(\varphi)=\int_{\Omega} g \delta(\varphi)|\nabla \varphi| d x d y
$$

And

$$
A_{g}(\varphi)=\int_{\Omega} g H(-\varphi) d x d y
$$

\section{Contour Evaluation}
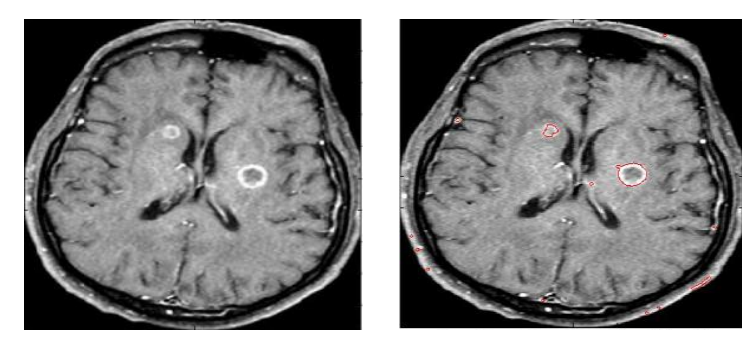

Fig. 4: Output image after applying level set method

This is very crucial to implicit active contours in the placement of the initial contour. Since the contour moves either inward or outward, its initial placement will determine the segmentation that is obtained. For example, if there is a single object in an image, an initial contour placed outside the object and propagated inward will segment the outer boundary of the object. However, if the object has a hole in the middle, it will not be possible to obtain the boundary of this hole unless the initial contour is placed inside the hole and propagated outward. It should be noted that more than one closed curve can be used for initialization of the zero-th level set. 


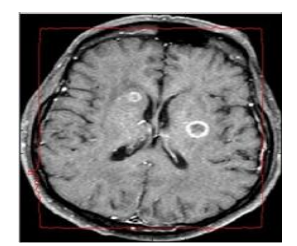

60 Iteration

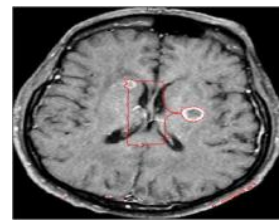

480 Iteration

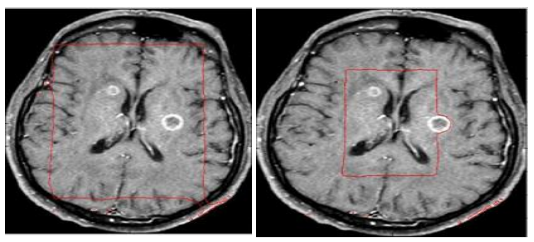

200 Iteration

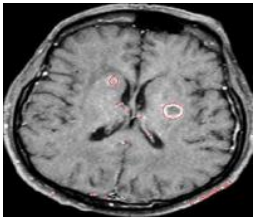

580 Iteration

Fig. 5: Contour Evaluation

First image in Fig. 5 shows the image after applying initial level set. Second image is after 200 iterations, next image after applying 360 iterations, then the image shown after iterate 480 times, next image is for image after applying 580 times. Last image shows that image after completing all the iterations.

\section{EXPERIMENTAL RESULTS}

The proposed method segmentation technique based on variational level set method has been applied to a variety of synthetic and real images in different modalities. The technique can also work well for other types of image such as CT image of human brain and PET image of human body.

The size for PET image considered here is $272 \times 307$. For PET image, a threshold value of 45 is chosen. After applying thresholding technique, a clear image is obtained as output that has no unexpected part. For this reason, any erosion technique is not used for this image. It directly applies level set technique on the original image. After 620 iterations, final image has been obtained (Fig. 6). Although the image is too large, it didn't take lots of time for evaluation.

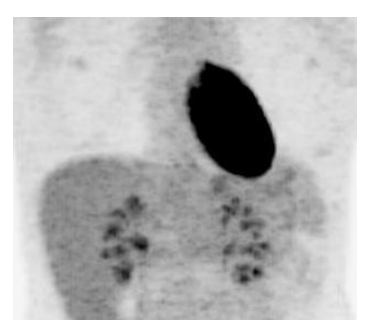

Fig. 6: PET image input and after applying proposed method

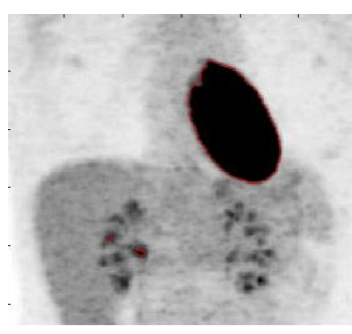

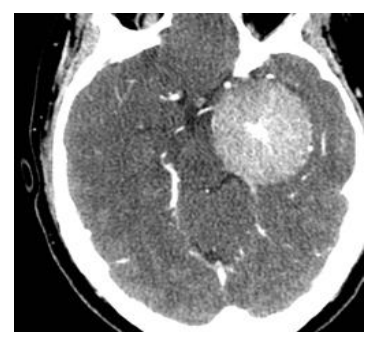

Fig. 7: CT image input and after applying proposed method

CT image that is considered here is smaller than the PET image. The CT image of human brain is used for experiment. The size of the CT image considered is 256x256. For CT image, a threshold value between 180 and 220 is used. After applying thresholding technique, it didn't get any clear image that has no unexpected part. Instead, it got some small fragments as output. For this reason, it requires to use erosion technique to remove these fragments. Then variational level set technique has been applied on the original image. After 550 iterations, final image is obtained (Fig. 7). Although the image is too large, it didn't take lots of time for evaluation.

\section{COMPARATIVE STUDY}

The active contour method is one of the most successful image segmentation techniques. It has received a tremendous amount of attention in medical image processing. The segmentation operation can be carried out manually or automatically. A manual segmentation requires a skilled operator trained to use a digital tool to mark the contours of the desired structures. The main objective of this paper is to select any better image processing algorithm than others among all the existing methods. Next objective is to segment specific object of image by modified existing methods. For this reason, variational level set method is selected without reinitialization that is totally different and more convenient than other traditional level set methods. Despite this method is more convenient to segment image and provide excellent for single object, but in case of detection of specific objects this method suffers for several shortcomings. First of all, this technique failed to detect specific object for more complex medical images. It works by considering whole image as single object as images has its own boundary. 

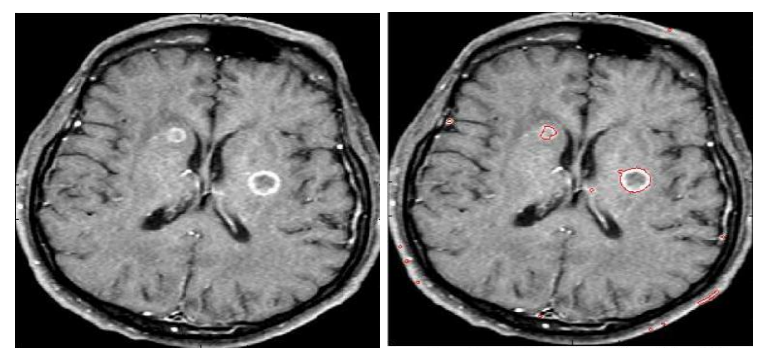

Fig. 8: Original MRI image and image after applying proposed method.
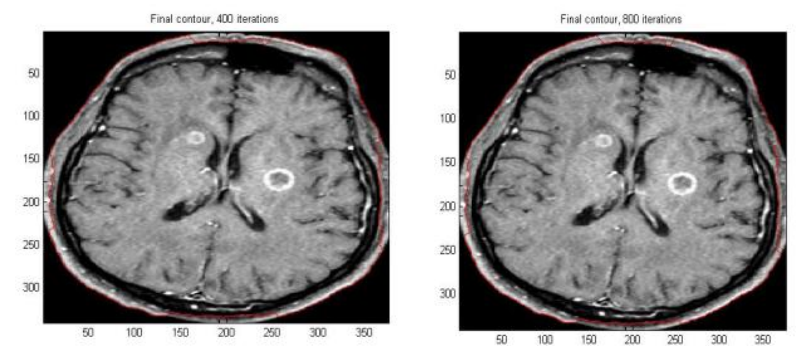

Fig. 9: Image after applying variational level set method without re-initialization.

To make the comparison process simpler, same images that already used for the proposed method for variational level set technique have been considered. This is the MRI image of human brain (Fig. 8). This image is almost complex than others. It contains two defected parts inside the image. But variational level set method is failed to select these objects (Fig. 9). The contour can't enter into the image boundary. But this method can detect or segment the objects if the image is simple or the image contain only single object. Fig. 10 demonstrates that the variational level set method works better for small and comparably simple image or image of single type objects.
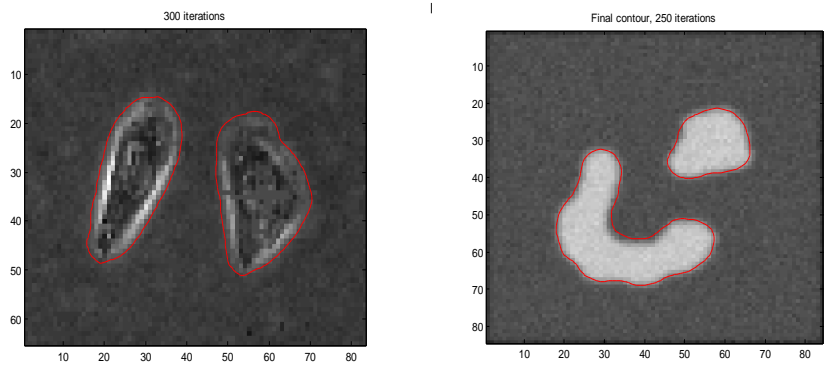

Fig. 10: Images after applying variational level set method.

\section{VII.}

\section{CONCLUSION}

The level set method plays vital role in modern computer vision research, image processing and analysis. The variational level set method is one of latest approach that is widely used in image processing. This paper has proposed segmentation technique based on variational level set method and some sort of existing technique specially thresholding. The main purpose was to segment specific object from different medical images like as MRI, CT, PET etc. Although the proposed method provides some remarkable opportunities to segment specific object from these images, but it has some limitations, such as it depends on defected object's intensity, very lengthy procedure, and high complexity in case of very large image. It can also be cited that this method is not fully automated as it is implemented in MATLAB. The threshold value is selected manually and better selection of threshold value gives better output. It also skips the automation process of iteration completion to reduce complexity. But the iteration process can also be implemented automatically for giving better support to the user and to make the process more convenient.

\section{REFERENCES}

[1] Rafael C. Gonzalez and Richard E. Woods, Digital Image Processing, $3^{\text {rd }}$ edition, Published by Pearson Prentice Hall, 2009.

[2] S.K. Weeratunga and C. Kamath, An Investigation of Implicit Active Contours for Scientific Image Segmentation, Visual Communications and Image Processing Conference, IS\&T/SPIE Symposium Electronic Imaging, San Jose, CA, January 18-22, 2004

[3] M. Khelif, F. Derraz and M. Beldagham, Application of Active Contour Models in Medical Image Segmentation.

[4] M. Droskey et al, An adaptive level set method for medical image segmentation.

[5] J. A. Sethian, Level Set Methods: An Act of Violence, Evolving Interfaces in Geometry, Fluid Mechanics, Computer Vision and Material Science.

[6] Tony F. Chan and Luminita A. Vese, Active Contours Without Edges, IEEE Transaction On Image Processing, Vol. 10, No. 2, FEBRUARY 2001

[7] V. Caselles, F. Catte, T. Coll, and F. Dibos, A geometric model for active contours in image processing, Numer. Math., Vol. 66, pp. 1-31, 1993 .

[8] M. Airouche et al, Image Segmentation Using Active Contour Model and Level Set Method Applied to Detect Oil Spills, Proceedings of the World Congress on Engineering, Vol. I, July 1-3, 2009, London, U.K.

[9] M. Kass, A.Witkin, and D. Terzopoulos, Snakes: Active contour models, Int'1 J. Comp. Vis., Vol. 1, pp. $321-331,1987$.

[10] Chunming Li et al, Level Set Evolution Without Re-initialization: A New Variational Formulation. Proceedings of the 2005 IEEE Computer Society Conference on Computer Vision and Pattern Recognition (CVPR'05) 
[11] P.K. Sahoo, S. Soltani, and A.K.C. Wong., A survey of thresholding techniques, Computer Vision Graph Image Proc., Vol. 41, pp.233-260, 1988.

[12] Thomas Brox and Joachim Weickert, Level set segmentation with multiple regions, IEEE Trans. Image Processing, Vol. 15, No. 9, pp. 3213-3218, 2006.

[13] H. Zhao, T. Chan, B. Merriman, and S. Osher, A variational level set approach to multiphase motion, J. Comp. Phys., Vol. 127, pp. 179-195, 1996.

Md. Golam Moazzam completed his B.Sc (Hons) in Electronics and Computer Science from Jahangirnagar University in 1997 and MS in Computer Science and Engineering from the same University in 2001, respectively. He is now an Associate Professor in the Dept. of Computer Science and Engineering, Jahangirnagar University, Dhaka-1342, Bangladesh.

Amita Chakraborty received her B.Sc. (Hons) in Electronics and Computer Science and MS in Computer Science and Engineering from Jahangirnagar University in 1997 and 2002 respectively. She is now an Assistant Professor in the Dept. of Computer Science and Engineering, Shaikh Burhanuddin College, Dhaka, Bangladesh.

Shamima Nasrin received her B.Sc. (Hons) in Electronics and Computer Science and MS in Computer Science and Engineering from Jahangirnagar University in 1998 and 2002 respectively. She is now a Lecturer in the Dept. of Computer Science and Engineering, Shaikh Burhanuddin College, Dhaka, Bangladesh.

Mohammad Selim is currently working as a Lecturer in the Department of Computer Science and Engineering, Shaikh Burhanuddin College, Dhaka, Bangladesh. 